\title{
The MIKE/HIRES Lyman limit survey: first results
}

\author{
Gabriel E. Prochter ${ }^{1}$, Jason X. Prochaska ${ }^{1}$, Scott M. Burles ${ }^{2}$, John \\ O'Meara ${ }^{2}$, and Rebecca A. Bernstein ${ }^{3}$ \\ ${ }^{1}$ UCSC Department of Astronomy and Astrophysics, UCO/Lick Observatory \\ ${ }^{2}$ Center for Space Research, Massachusetts Institute of Technology \\ ${ }^{3}$ Department of Astronomy, University of Michigan
}

\begin{abstract}
We present preliminary results of a detailed analysis of the $z=3.55$ Lyman limit system observed in the optical spectrum of Q2000-330. We have fitted Voigt profiles to the system to determine the column densities and Doppler parameters of its many components. For its major components we have also determined the ionisation fraction of the gas and obtained crude estimates of metallicities. We find order of magnitude variations in ionisation fraction and metallicity in components separated by as little as $150 \mathrm{~km} \mathrm{~s}^{-1}$ in velocity space. This work is presented as an example of a campaign we have begun to collect such information on a sample of $\sim 100$ such systems in an effort to accurately characterise this enigmatic and important class of quasar absorption line systems.
\end{abstract}

\section{Introduction to LLS survey}

Bridging the gap between very high HI column density systems (the damped Ly $\alpha$ systems, DLAs) and very low HI column density (the Ly $\alpha$ forest), Lyman limit systems (LLS) are currently the most poorly understood major class of quasar absorption line systems. To address this dearth of observational analysis, we have begun a programme to collect $\sim 100$ high resolution spectra of LLSs in the redshift range $2.6<z<4.0$.

\section{Scientific goals}

The main scientific goals of this programme are:

- A census of metals in the QAL systems which may dominate the metal content of the Universe;

- The ionisation state of an entire class of systems with partially or highly ionised gas. This analysis should reveal important clues to the nature and intensity of the extragalactic UV background at $z>2$;

- Resolved velocity fields which describe the kinematic characteristics and therefore the physical nature of these galaxies (e.g. winds, mini-halos);

- Crude relative chemical abundances which relate to the nucleosynthetic history and dust depletion levels of the gas;

- The frequency distribution of HI column densities in the interval $N(\mathrm{HI})=10^{17}-$ $10^{20} \mathrm{~cm}^{-2}$. 


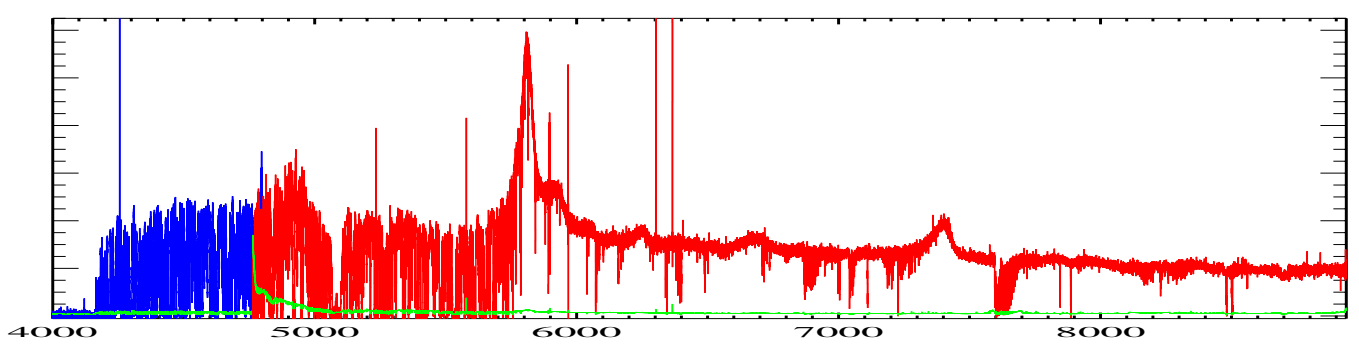

Figure 1. MIKE blue and red spectra of Q2000-330. The left of this spectrum clearly displays the signature of a Lyman Limit System. This represents 2400s of data collection on the MIKE spectrograph. The data was reduced using an IDL pipeline written by members of this collaboration. The resolution of the data is FWHM $\sim 10 \mathrm{~km} \mathrm{~s}^{-1}$ on the blue side and $\sim 12 \mathrm{~km} \mathrm{~s}^{-1}$ on the red.
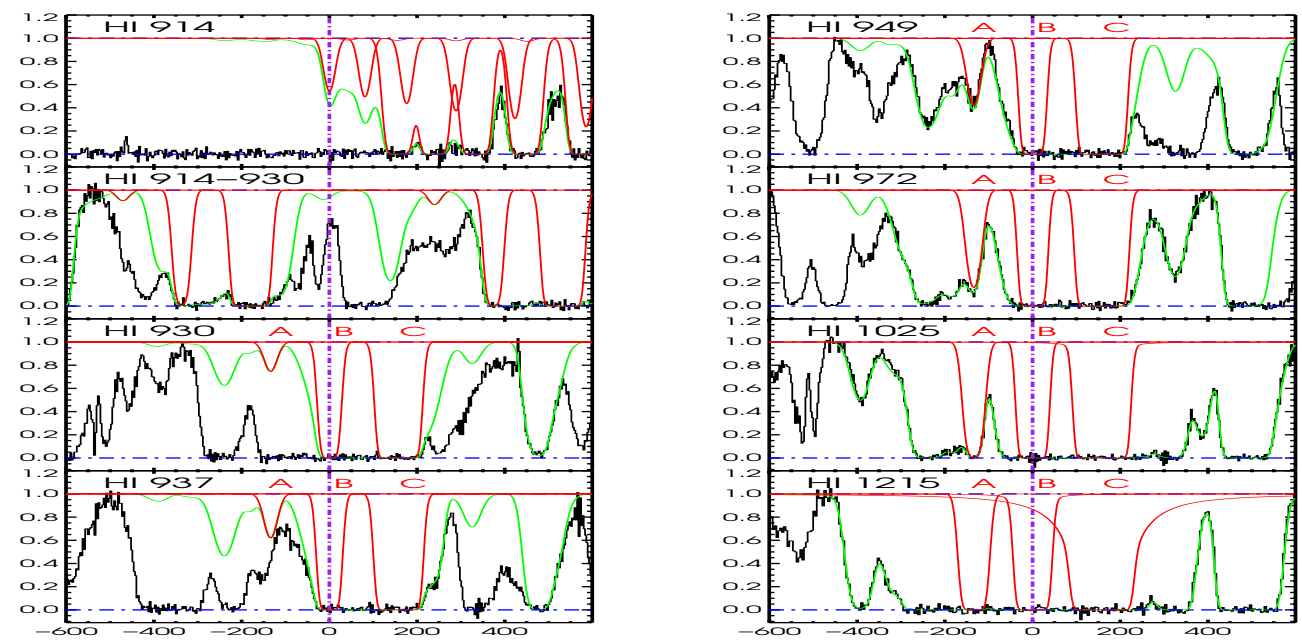

Figure 2. VPFIT Voigt best-fit profiles to the HI absorption. The red curves are individual fits to the three components, labelled here and in Fig. 3 as A, B, and C, which correspond to metallic absorption associated with this LLS. The green curve is our total fit, which includes many components that are not obviously associated with any metallic absorption. These fits were done simultaneously with the metal absorption profiles depicted in Fig. 3.

\section{Example system: Q2000-330}

\subsection{Data and analysis}

Fig. 1 presents blue and red spectra of Q2000-330 collected in 2400s on the MIKE echelle spectrograph at the Magellan Telescope. This is a $z=3.77$ quasar, with an obvious Ly $\alpha$ forest, which complicates the fitting of a Voigt profile to the LLS detected at $z=3.55$.

Figs. 2 and 3 present the HI and metal features along with VPFIT best-fit Voigt profiles. With this data we then turned to CLOUDY and determined the ionisation fraction for components $\mathrm{B}$ and $\mathrm{C}$, assuming a Haardt \& Madau spectrum at $z=3.5$. The results of the VPFIT and CLOUDY analyses are summarised in the table.

\subsection{Results}

VPFIT and CLOUDY results are summarised in the following table. The values for $(\mathrm{HI} / \mathrm{H})$ and $(\mathrm{OI} / \mathrm{O})$ are inferred based primarily on the OI/SiII and OI/CII ratios for each system (where available). 

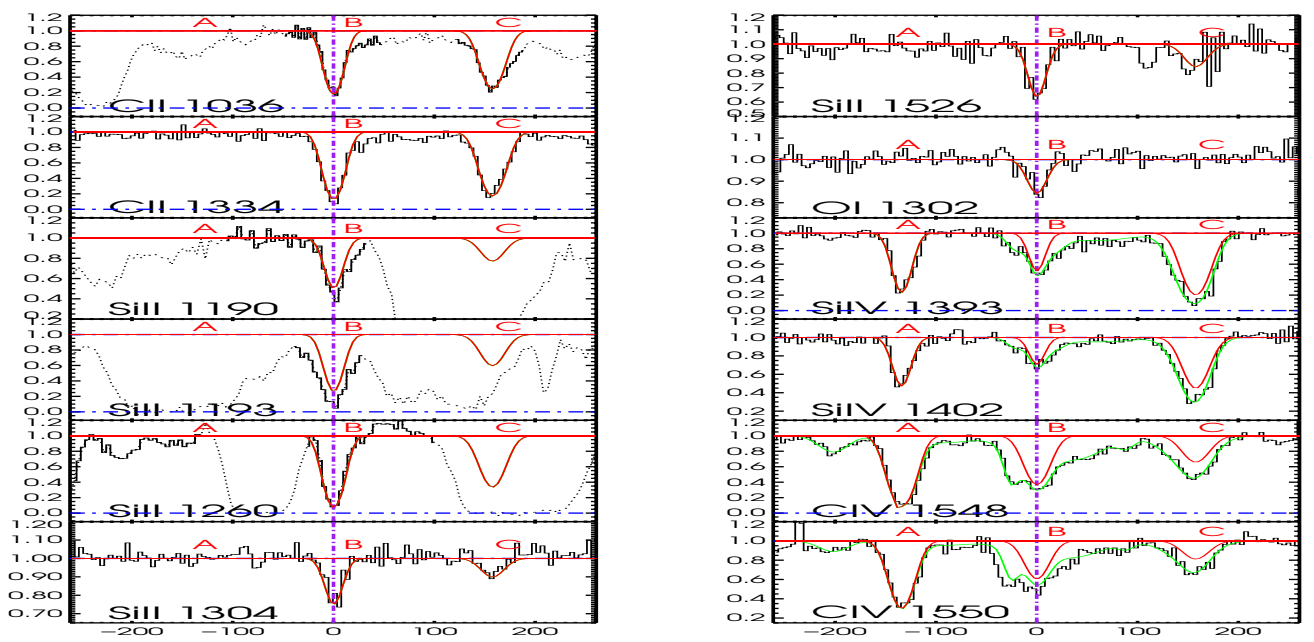

Figure 3. VPFIT Voigt profile fits to metal absorption associated with this LLS. Again, the red curves correspond to individual components which we tied to specific components in the HI absorption and are labelled as in Fig. 2. The table below contains the VPFIT analysis results for these components, as well as estimates of ionisation fraction and metallicity.

\begin{tabular}{cccc}
\hline & $\mathrm{A}$ & $\mathrm{B}$ & $\mathrm{C}$ \\
\hline $\mathrm{z}$ & 3.5479 & 3.5499 & 3.5523 \\
$\log \mathrm{N}(\mathrm{HI})$ & 14.936 & 16.589 & 18.122 \\
& $( \pm 0.041)$ & $( \pm 0.055)$ & $( \pm 0.180)$ \\
$\log \mathrm{N}(\mathrm{CIV})$ & 13.94 & 13.57 & 13.23 \\
& $( \pm 0.039)$ & $( \pm 0.228)$ & $( \pm 0.973)$ \\
$\mathrm{b}(\mathrm{km} / \mathrm{s})$ & 18.79 & 16.03 & 21.97 \\
$v_{i}(\mathrm{~km} / \mathrm{s})$ & -135 & 0 & 150 \\
{$[\mathrm{OI} / \mathrm{HI}]$} & $<1.5$ & 0.13 & $<-1.68$ \\
{$[\mathrm{OI} / \mathrm{SiI}]$} & $<-0.85$ & -1.05 & -1.05 \\
{$[\mathrm{OI} / \mathrm{CII}]$} & $<0.03$ & -0.93 & -1.20 \\
$(\mathrm{HI} / \mathrm{H})$ & 0.012 & 0.03 & 0.005 \\
$(\mathrm{OI} / \mathrm{O})$ & 0.003 & 0.02 & 0.0005 \\
\hline
\end{tabular}

\section{Summary}

- We have begun a survey of $\sim 100$ LLSs in the redshift range $2.6<z<4.0$ to characterise this poorly understood class of quasar absorption system.

- Preliminary results from one such system indicates that LLS are highly complicated, with a wide range of metallicities and ionisation fractions, indicating that a complete understanding of these systems will require a much larger high-resolution sample of them than currently exists.

- The detailed small-scale variations detected in this system raise interesting questions about feedback, star-formation, and galaxy evolution in this early epoch, and may also have important implications for the DLA class of absorbers, where such variations are difficult to measure.

- The magnitude of the variation over small jumps in velocity space imply a highly dynamic environment. Our completed sample will allow us to study this sort of environment in detail and determine what role it plays in the evolution of the Universe as a whole 